University of Wollongong

Research Online

Australian Institute for Innovative Materials -

Papers

Australian Institute for Innovative Materials

$1-1-2018$

Coercivity degradation caused by inhomogeneous grain boundaries in sintered Nd-Fe-B permanent magnets

Hansheng Chen

University of Sydney

Fan Yun

University of Sydney

Jiangtao Qu

University of Sydney

Yingfei Li

University of Wollongong

Zhenxiang Cheng

University of Wollongong, cheng@uow.edu.au

See next page for additional authors

Follow this and additional works at: https://ro.uow.edu.au/aiimpapers

Part of the Engineering Commons, and the Physical Sciences and Mathematics Commons

Research Online is the open access institutional repository for the University of Wollongong. For further information contact the UOW Library: research-pubs@uow.edu.au 


\title{
Coercivity degradation caused by inhomogeneous grain boundaries in sintered Nd-Fe-B permanent magnets
}

\author{
Abstract \\ Quantitative correlation between intrinsic coercivity and grain boundaries in three dimensions is critical to \\ further improve the performance of sintered Nd-Fe-B permanent magnets. Here, we quantitatively reveal \\ the local composition variation across and especially along grain boundaries using the powerful atomic- \\ scale analysis technique known as atom probe tomography. We also estimate the saturation \\ magnetization, magnetocrystalline anisotropy constant, and exchange stiffness of the grain boundaries \\ on the basis of the experimentally determined structure and composition. Finally, using micromagnetic \\ simulations, we quantify the intrinsic coercivity degradation caused by inhomogeneous grain boundaries. \\ This approach can be applied to other magnetic materials for the analysis and optimization of magnetic \\ properties.

\section{Disciplines} \\ Engineering | Physical Sciences and Mathematics

\section{Publication Details} \\ Chen, H., Yun, F., Qu, J., Li, Y., Cheng, Z., Fang, R., Ye, Z., Ringer, S. P. \& Zheng, R. (2018). Coercivity \\ degradation caused by inhomogeneous grain boundaries in sintered Nd-Fe-B permanent magnets. \\ Physical Review Materials, 2 (5), 054404-1-054404-9.

\section{Authors} \\ Hansheng Chen, Fan Yun, Jiangtao Qu, Yingfei Li, Zhenxiang Cheng, Ruhao Fang, Zhixiao Ye, Simon Peter \\ Ringer, and Rongkun Zheng
}




\title{
Coercivity degradation caused by inhomogeneous grain boundaries in sintered Nd-Fe-B permanent magnets
}

\author{
Hansheng Chen, ${ }^{1,2,3}$ Fan Yun, ${ }^{1,2,3}$ Jiangtao Qu, ${ }^{1,2,3}$ Yingfei Li, ${ }^{4}$ Zhenxiang Cheng, ${ }^{4}$ Ruhao Fang, ${ }^{1,2,3}$ Zhixiao Ye, \\ Simon P. Ringer, ${ }^{2,6}$ and Rongkun Zheng, ${ }^{1,2,3, *}$ \\ ${ }^{1}$ School of Physics, The University of Sydney, Sydney, NSW, 2006, Australia \\ ${ }^{2}$ The University of Sydney Nano Institute, The University of Sydney, Sydney, NSW 2006, Australia \\ ${ }^{3}$ Australian Centre for Microscopy and Microanalysis, The University of Sydney, Sydney, NSW, 2006, Australia \\ ${ }^{4}$ Institute for Superconducting and Electronic Materials, University of Wollongong, New South Wales 2522, Australia \\ ${ }^{5}$ Hengdian Group DMEGC Magnetics Co. Ltd., Zhejiang, 322118, China \\ ${ }^{6}$ School of Aerospace, Mechanical and Mechatronic Engineering, The University of Sydney, Sydney, NSW 2006, Australia
}

(Received 5 October 2017; published 14 May 2018)

\begin{abstract}
Quantitative correlation between intrinsic coercivity and grain boundaries in three dimensions is critical to further improve the performance of sintered $\mathrm{Nd}-\mathrm{Fe}-\mathrm{B}$ permanent magnets. Here, we quantitatively reveal the local composition variation across and especially along grain boundaries using the powerful atomic-scale analysis technique known as atom probe tomography. We also estimate the saturation magnetization, magnetocrystalline anisotropy constant, and exchange stiffness of the grain boundaries on the basis of the experimentally determined structure and composition. Finally, using micromagnetic simulations, we quantify the intrinsic coercivity degradation caused by inhomogeneous grain boundaries. This approach can be applied to other magnetic materials for the analysis and optimization of magnetic properties.
\end{abstract}

DOI: 10.1103/PhysRevMaterials.2.054404

\section{INTRODUCTION}

Rare-earth (RE) elements as strategic materials have increasingly attracted attention due to their catalytic effects on the functionalization of materials for use in various fields such as aviation, marine, spacecraft, hybrid vehicles, energy, microelectronics, national defense, and others [1-3]. Specifically, owing to their extraordinary magnetic properties, sintered $\mathrm{Nd}-\mathrm{Fe}-\mathrm{B}$ permanent magnets have been broadly integrated into various applications, including advanced electric motor technologies, which partly relieve the current worldwide energy crisis [3]. However, the intrinsic coercivity $\left(H_{\mathrm{ci}}\right)$ of sintered $\mathrm{Nd}-\mathrm{Fe}-\mathrm{B}$ permanent magnets is still only $\sim 20-30 \%$ of the theoretical Stoner-Wolfarth limit (Brown's paradox) [3-5]. The $H_{\mathrm{ci}}$ is primarily determined by the nucleation field of reverse magnetic domains near grain boundaries (GBs), and the three-dimensional (3D) quantitative correlation between $H_{\mathrm{ci}}$ and GBs is the key to further improving the $H_{\mathrm{ci}}$ of sintered Nd-Fe-B permanent magnets [6].

The first step is to qualitatively understand the effects of GBs on the $H_{\mathrm{ci}}$. Sasaki et al. showed that dopant Ga atoms cause the formation of thick and nonmagnetic GBs, leading to coercivity enhancement in sintered Nd-Fe-B permanent magnets [7]. Li et al. reported that the addition of $\mathrm{Cu}$ triggers the formation of continuous $\mathrm{GBs}$ and $\mathrm{Cu}$-rich thin layers between the matrix grains and Nd-rich phases, resulting in an approximately threefold coercivity enhancement in $\mathrm{Cu}-$ doped sintered Nd-Fe-B permanent magnets [8]. Nonetheless, quantifying the composition of GBs in $3 \mathrm{D}$ at the atomic scale

\footnotetext{
*Corresponding author: rongkun.zheng@ sydney.edu.au
}

remains challenging, which impedes the precise interpretation of the role of GBs.

As a novel technique, atom probe tomography (APT) detects individual atoms in $3 \mathrm{D}$ at the atomic scale $[9,10]$. In laser-assisted APT, individual atoms (or groups of atoms) are evaporated from a needle-shaped tip with a diameter up to $\sim 100 \mathrm{~nm}$ by applying a voltage as high as $\sim 10,000 \mathrm{~V}$ and picosecond laser pulses. 3D reconstruction of the fieldevaporated volume is achieved based on the evaporation sequence and time-of-flight mass spectrometry. According to Sepehri-Amin et al., some GBs in sintered Nd-Fe-B permanent magnets are made up of as much as $\sim 65$ at. \% ferromagnetic elements $(\mathrm{Fe}+\mathrm{Co})$, indicating that these GBs may be ferromagnetic or paramagnetic rather than nonmagnetic [11]. Sepehri-Amin et al. have also reported that thin layers with $\sim 2$ at. $\% \mathrm{Cu}$ between the matrix grains and Nd-rich GBs decouple the neighboring matrix grains and hence increase the $H_{\mathrm{ci}}$ [11]. Furthermore, Dy-substituted sintered Nd-Fe-B permanent magnets have also been intensively studied using APT [12-14]. Sepehri-Amin et al. showed that nearly pure $\mathrm{Nd}$ layers of $\sim 6-\mathrm{nm}$ thickness between the $(\mathrm{Nd}, \mathrm{Dy})_{2} \mathrm{Fe}_{14} \mathrm{~B}$ matrix grains and the (Nd,Dy)-rich phase led to coercivity enhancement [12,13]. In Dy-substituted Nd-Fe-B permanent magnets processed by the grain-boundary diffusion process, a high Dy concentration of $\sim 3.3$ at. $\%$ in the $(\mathrm{Nd}, \mathrm{Dy})_{2} \mathrm{Fe}_{14} \mathrm{~B}$ shell increases the magnetocrystalline anisotropy field and $H_{\mathrm{ci}}$ [14].

To fully quantify the correlation between the $H_{\mathrm{ci}}$ and $\mathrm{GBs}$ in sintered Nd-Fe-B permanent magnets, researchers have devoted considerable effort to micromagnetic simulations [5,15-19]. Schrefl et al. reported that long-range magnetostatic interactions between neighboring matrix grains across the GB 
are trivial for reducing the $H_{\mathrm{ci}}$ [15]. According to Hrkac et al., a 1.6-nm-thick distorted region with decreased magnetic anisotropy led to a reduction of the $H_{\mathrm{ci}}$ from 4.4 to $2.8 \mathrm{~T}$ in the single-grain model $[16,17]$. Ma et al. reported that ferromagnetic GBs with a saturation magnetization $\left(M_{\mathrm{S}}\right)$ of $0.8 \mathrm{~T}$ led to an $\sim 9 \% H_{\mathrm{ci}}$ reduction compared to nonmagnetic GBs with a $M_{\mathrm{S}}$ of $0 \mathrm{~T}$ in multiple-main-phase Nd-Ce-Fe-B magnets [19]. However, the aforementioned micromagnetic simulations suffer two major shortcomings: (1) the parameter estimation deployed in micromagnetic simulations was not based on experimental observations, and (2) only cross-GB variation was considered, whereas the effects of composition variation along the GB were overlooked.

In this paper, we quantitatively investigate local composition variation across and especially along the GB in 3D by scanning electron microscopy-electron backscatter diffraction (SEM-EBSD) and atom probe tomography. We also bridge experiments and theories by estimating the $M_{\mathrm{S}}$, magnetocrystalline anisotropy constant $\left(K_{1}\right)$, and exchange stiffness $(A)$ of the GBs based on experimental observations. Finally, using micromagnetic simulations, we precisely quantify the $H_{\mathrm{ci}}$ degradation caused by inhomogeneous GBs in sintered Nd$\mathrm{Fe}-\mathrm{B}$ permanent magnets.

\section{EXPERIMENTAL SECTION}

\section{A. Material}

The nominal composition of the sintered Nd-Fe-B permanent magnet is listed in Supplemental Material, Table SI [20]. The sample was manufactured by traditional procedures of powder metallurgy, including induction melting, strip casting, hydrogen decrepitation, jet milling, isostatic pressing, sintering $(\sim 1358 \mathrm{~K}$ for $\sim 4 \mathrm{~h})$, and postsinter annealing $(\sim 1173$ and $\sim 893 \mathrm{~K}$ for $\sim 2$ and $\sim 4 \mathrm{~h}$, respectively).

\section{B. Magnetic hysteresis loops at different temperatures}

Magnetic hysteresis loops of the sintered Nd-Fe-B permanent magnet were recorded from -7 to $7 \mathrm{~T}$ at $280,300,320$, 340 , and $360 \mathrm{~K}$ using a vibrating sample magnetometer in a Quantum Design physical property measurement system.

\section{Microstructural characterization}

SEM mapping was investigated in a Zeiss Ultra Plus fieldemission scanning electron microscope. Kikuchi patterns were recorded with an acceleration voltage of $25 \mathrm{kV}$ and an aperture size of $120 \mu \mathrm{m}$ and in "high current" mode. The step size was selected as $0.5 \mu \mathrm{m}$.

The sample for APT experiments was milled to a diameter of tens of nanometers using the tripod polishing method followed by the focused ion-beam (FIB) milling [21]. Figure S1 exhibits the workflow of the preparation of atom probe tips [20]. The sample was first mounted as shown in Fig. S1(a) [20]. The sample was then mechanically polished with a wedge on one side and mounted onto a copper grid. Note that the $\langle 001\rangle$ direction/ $c$ axis was nearly parallel to the tips. The sample was coarsely milled by SEM-FIB technique using Ga ions from 30 $\mathrm{kV}$ and $15 \mathrm{nA}$ to $30 \mathrm{kV}$ and $2 \mathrm{nA}$. Finally, the sample was annularly milled using Ga ions from $30 \mathrm{kV}$ and $250 \mathrm{pA}$ to $10 \mathrm{kV}$ and $200 \mathrm{pA}$ in the manner shown in Fig. S1(c) [20].

APT measurements were carried out in a picosecondpulse UV-laser-assisted CAMECA local electrode atom probe 4000X Si. The needle-shaped sample was subjected to a high vacuum at cryogenic temperatures under an intense electric field generated by a dc voltage. Ions evaporated from the specimen surface under the laser pulse were detected on the basis of their mass-to-charge ratios recorded by the timeof-flight spectroscopy. In our study, APT experiments were performed at $50 \mathrm{~K}$ with a 355 -nm-wavelength laser with a pulse energy of 50-70 pJ and a pulse frequency of 100 $200 \mathrm{kHz}$. Tomographic reconstructions were performed using CAMECA's Integrated Visualization and Analysis Software (IVAS) version 3.6.6 $[9,10]$.

\section{Micromagnetic simulations}

Figure S2(a) shows the $100-\mathrm{nm} \times 100-\mathrm{nm} \times 100-\mathrm{nm}$ sandwich model (matrix grain 1-GB-matrix grain 2) used to simulate the demagnetization curves of a sintered $\mathrm{Nd}-\mathrm{Fe}-$ $\mathrm{B}$ permanent magnet [20]. The model was discretized by cubic meshes with a size of $2 \mathrm{~nm}$, which is smaller than the exchange length $(\sim 2.77 \mathrm{~nm})$ and the domain wall width $(\sim 5.24 \mathrm{~nm})$ of $\mathrm{Nd}_{2} \mathrm{Fe}_{14} \mathrm{~B}$ matrix phases [22]. The LandauLifshitz-Gilbert equation at each node was calculated using the 3D NIST Object Oriented MicroMagnetic Framework (OOMMF) SOFTWARE [23]. Three key parameters deployed into the micromagnetic simulation are the $A$, which indicates the strength of Coulombic repulsion between two neighboring electrons (usually on neighboring atoms), $K_{1}$, and $M_{\mathrm{S}}$ of the materials [24]. The latter two parameters both determine the anisotropy field and theoretical $H_{\mathrm{ci}}$ of the materials [24]. Here, the $A, K_{1}$, and $M_{\mathrm{S}}$ of the matrix grains were selected to be $12.5 \mathrm{pJ} / \mathrm{m}, 4.5 \mathrm{MJ} / \mathrm{m}^{3}$, and $1.30 \mathrm{MA} / \mathrm{m}$, respectively [25]. The $A, K_{1}$, and $M_{\mathrm{S}}$ of 10 -nm-wide GBs with Fe compositions of $0,10,20,30,40,50,60$, and 67 at. \% (denoted by 0 , $10,20,30,40,50,60$, and $67 \mathrm{Fe}$, respectively) are listed in Table SII [20]. Figure S2 also shows the simulated model for an inhomogeneous GB with an average Fe composition of $\sim 38.7$ at. \%, where the concentration of $\mathrm{Fe}$ increases from 0 to 67 at. $\%$ gradually and then decreases to 50 at. $\%$ in $100 \mathrm{~nm}$ [20]. The corresponding parameters deployed for the micromagnetic simulations are also shown in Table SII [20]. The initial directions of the magnetization of MG1, MG2, and GB are upward, and the external field was applied downward from 0 to $6 \mathrm{~T}$.

\section{RESULTS AND DISCUSSION}

\section{A. Magnetic properties}

Figure 1 illustrates the magnetic properties of a sintered $\mathrm{Nd}-\mathrm{Fe}-\mathrm{B}$ permanent magnet at elevated temperatures. The $H_{\text {ci }}$ decreases from $1.80 \mathrm{~T}$ at $280 \mathrm{~K}$ to $0.83 \mathrm{~T}$ at $360 \mathrm{~K}$. The rapid increase, not plateau, of the initial magnetization curve confirms that the $H_{\mathrm{ci}}$ of the sintered Nd-Fe-B permanent magnet is mainly controlled by the nucleation of reverse magnetic domains rather than by the domain-wall pinning. 


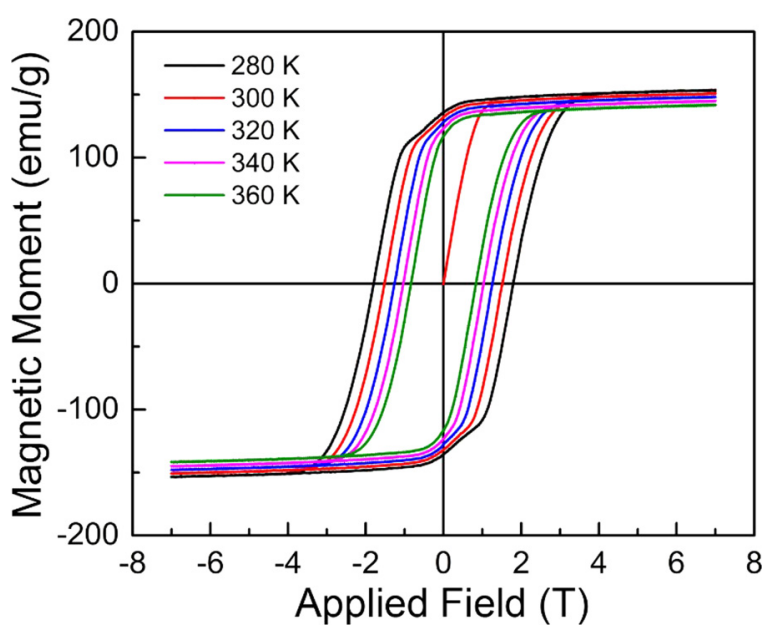

FIG. 1. Magnetization curves of a sintered Nd-Fe-B permanent magnet at 280, 300, 320, 340, and $360 \mathrm{~K}$.

\section{B. Microstructure/microchemistry observations}

The microstructural features, particularly the GBs, were examined using advanced microscopy techniques. The secondary electron image in Fig. 2(a) shows the surface topography of the sintered Nd-Fe-B permanent magnet, in which the $(\mathrm{Nd}, \mathrm{Pr})$-rich triple junctions and GBs were etched deeper, consistent with our previous work [26]. The corresponding backscattered electron and phase map images clearly demonstrate that $(\mathrm{Nd}, \mathrm{Pr})_{2}(\mathrm{Fe}, \mathrm{Co})_{14} \mathrm{~B}$ matrix grains [dark contrast in Fig. 2(b)] are surrounded by (Nd,Pr)-rich triple junctions and GBs [bright contrast in Fig. 2(b)].

To gain deeper insight into the microstructure/microchemistry of (Nd,Pr)-rich $\mathrm{GBs}$, we performed APT experiments on the same sample. Figure 3 displays an inhomogeneous GB and corresponding 3D atom distribution maps of $\mathrm{Fe}, \mathrm{Nd}, \mathrm{Pr}, \mathrm{B}, \mathrm{Co}, \mathrm{Cu}, \mathrm{Ga}$, and $\mathrm{Al}$ at the atomic scale. The quantitative compositions of the matrix grains and GBs were analyzed and summarized in Table I. The chemical composition of the matrix grains (MG1 and MG2) was determined to be $\mathrm{Nd}_{9.4} \mathrm{Pr}_{2.4} \mathrm{Fe}_{81.9} \mathrm{Co}_{0.6} \mathrm{~B}_{4.9} \mathrm{Al}_{0.4} \mathrm{Others}_{0.4}$. The ratio between $\mathrm{Nd}+\mathrm{Pr}$ and $\mathrm{Fe}+\mathrm{Co}$ in the matrix grains is $\sim 1: 7.0$. This ratio is consistent with that between $\mathrm{Nd}$ and $\mathrm{Fe}$ in $\mathrm{Nd}_{2} \mathrm{Fe}_{14} \mathrm{~B}$ phase, thus verifying the high accuracy of the APT results. Nevertheless, the concentration of B was determined to be $\sim 4.9$ at. \%, which was considerably less than the expected value $(\sim 5.8$ at. $\%)$. This difference may arise from the retention effects of $\mathrm{B}$ atoms in a high evaporation field $(64 \mathrm{~V} / \mathrm{nm})[16,27]$. The chemical composition of the GB was determined to be $\mathrm{Nd}_{30.8} \operatorname{Pr}_{13.4} \mathrm{Fe}_{42.0} \mathrm{Co}_{6.4} \mathrm{Cu}_{4.0} \mathrm{Ga}_{0.2} \mathrm{~B}_{2.3}$ Others ${ }_{0.9}$, suggesting that $\mathrm{Nd}, \mathrm{Pr}, \mathrm{Co}, \mathrm{Cu}$, and $\mathrm{Ga}$ atoms were enriched at the $\mathrm{GB}$, whereas $\mathrm{Al}, \mathrm{B}$, and $\mathrm{Fe}$ atoms were depleted. For the alloying additives, $\mathrm{Co}, \mathrm{Cu}$, and $\mathrm{Ga}$ atoms tend to aggregate along the interfaces between the (Nd,Pr)-rich $\mathrm{GB}$ and $(\mathrm{Nd}, \mathrm{Pr})_{2}(\mathrm{Fe}, \mathrm{Co})_{14} \mathrm{~B}$ matrix grains and form thin layers $(\sim 7 \mathrm{~nm}$ for Co atoms, $\sim 5 \mathrm{~nm}$ for $\mathrm{Cu}$ atoms, and $\sim 3 \mathrm{~nm}$ for Ga atoms, as shown in Fig. S3 [20]). What is astonishing is that $\mathrm{Fe}, \mathrm{Nd}$, and $\mathrm{Pr}$ atoms are not uniformly distributed along the (Nd,Pr)-rich GB.

In detail, Fig. 4 shows the enlarged 3D atom distribution map of $\mathrm{Fe}$ and the corresponding concentration profiles of $\mathrm{Fe}, \mathrm{Nd}$, and Pr. The lowest concentration of RE elements ( $\sim 25$ at. \% for $\mathrm{Nd}$ and $\sim 10$ at. \% for Pr) and highest concentration of $\mathrm{Fe}(\sim 50$ at. \%) were observed in the relatively thick region of the GB $(\sim 15 \mathrm{~nm})$, as marked by the red box. Along the GB, a high concentration of RE elements ( $\sim 50$ at. $\%$ for $\mathrm{Nd}$ and $\sim 25$ at. \% for $\mathrm{Pr})$ and a low concentration of $\mathrm{Fe}(\sim 10$ at. \%) were observed in the relatively thin region of the $\mathrm{GB}(\sim 7 \mathrm{~nm})$, as marked by the blue box. More precisely, 1D concentration profiles extracted along the GB [Fig. 4(c)] reveal the variation of Fe (from $\sim 60$ to $\sim 3$ at. \%), Nd (from $\sim 20$ to $\sim 57$ at. $\%$ ), and $\operatorname{Pr}$ (from $\sim 7$ to $\sim 25$ at. \%) within $\sim 70 \mathrm{~nm}$. Meanwhile, the concentration of Co remains $\sim 7$ at. \% in the entire GB (Fig. S3) [20]. Hence, the gross concentration of ferromagnetic elements (Fe and $\mathrm{Co}$ ) varies dramatically from $\sim 67$ to $\sim 10$ at. \% within $\sim 70 \mathrm{~nm}$ along the GB, implying substantial variation of the magnetic behaviors (such as $A, K_{1}$, and $M_{\mathrm{S}}$ ) along the GB.

\section{Parameter estimation for micromagnetic simulations}

The microstructural features revealed by APT enable the precise estimation of the $M_{S}, K_{1}, A$ of the GBs with various compositions, and therefore more reliable micromagnetic simulations. Although numerous TEM studies have shown crystalline structures of GBs in sintered Nd-Fe-B permanent magnets, inconsistencies exist in the literature because Nd-rich GBs are easily oxidized during SEM and TEM specimen preparation [28-32]. Previously, thin Nd-rich GBs were found to be amorphous in sintered Nd-Fe-B permanent magnets $[28,30]$. As GBs broadened, a face-centered cubic (fcc) structure was observed [28]. In addition to the width of GBs, the composition of GBs also affects their crystal structures. As the $\mathrm{O}$ content increases, crystalline structures of Nd-rich GBs change from double-hexagonal close-packed to fcc and hexagonal close-packed [29]. Recent

TABLE I. Chemical compositions of the matrix grains and GB (where the concentration of Nd is greater than 13.9 at. \%), as determined by APT.

\begin{tabular}{|c|c|c|c|c|c|}
\hline at. $\%$ & $\mathrm{Fe}$ & $\mathrm{Nd}$ & $\operatorname{Pr}$ & $\mathrm{Co}$ & $\mathrm{Cu}$ \\
\hline MG & $81.923 \pm 0.035$ & $9.358 \pm 0.009$ & $2.418 \pm 0.004$ & $0.557 \pm 0.002$ & $0.025 \pm 0.000$ \\
\hline GB & $42.013 \pm 0.066$ & $30.785 \pm 0.054$ & $13.351 \pm 0.033$ & $6.395 \pm 0.022$ & $3.953 \pm 0.017$ \\
\hline at. $\%$ & $\mathrm{Ga}$ & B & $\mathrm{Al}$ & Others & \\
\hline MG & $0.012 \pm 0.000$ & $4.899 \pm 0.006$ & $0.409 \pm 0.002$ & $0.399 \pm 0.004$ & \\
\hline GB & $0.159 \pm 0.003$ & $2.335 \pm 0.013$ & $0.110 \pm 0.003$ & $0.899 \pm 0.017$ & \\
\hline
\end{tabular}



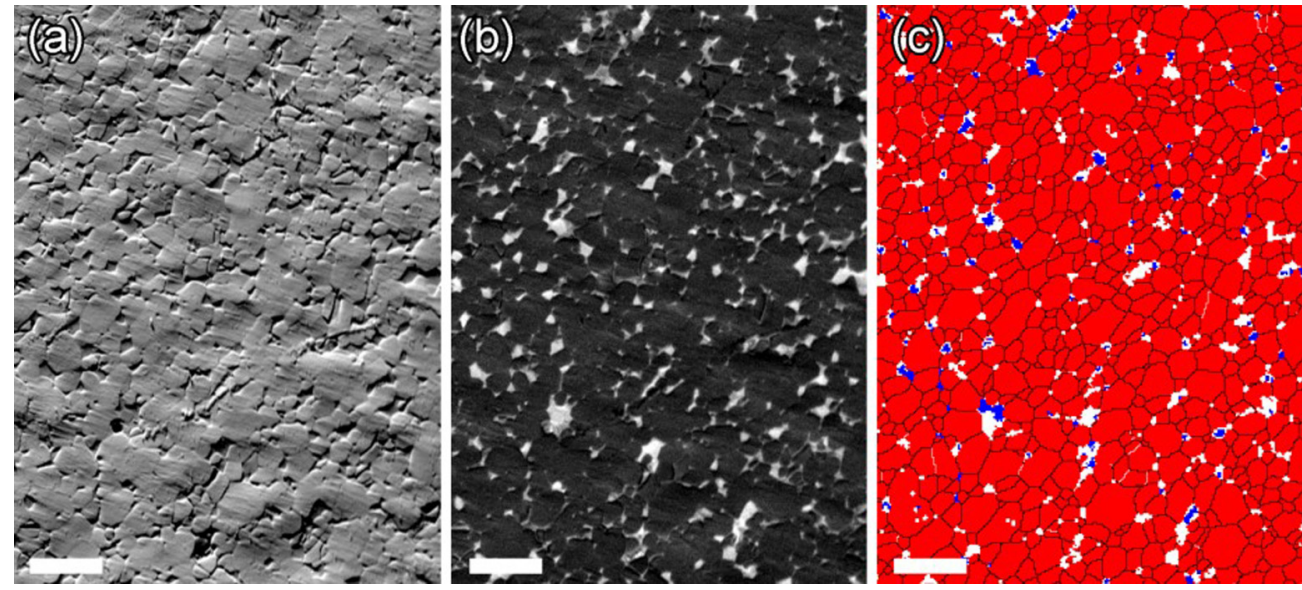

FIG. 2. SEM-EBSD analysis of a sintered Nd-Fe-B permanent magnet. (a) Secondary electron image. (b) Backscattered electron image. (c) Phase map of the sintered Nd-Fe-B permanent magnet, in which the matrix grains are indexed in red and the (Nd,Pr)-rich phase is indexed in blue. The unindexed regions are white. The scale bars are $20 \mu \mathrm{m}$.

TEM and APT observations have also shown the composition of RE elements such as $\mathrm{Nd}$ and $\mathrm{Pr}$ may affect crystalline structures of GBs in sintered $\mathrm{Nd}-\mathrm{Fe}-\mathrm{B}$ permanent magnets [11,31,32]. Sasaki et al. reported that the crystalline Nd-rich
GB contains a greater amount of $\mathrm{Nd}(\sim 64.7$ at. $\%)$ and a lower concentration of $\mathrm{Fe}(\sim 30$ at. $\%)$, while an amorphous Nd-rich GB contains a lower amount of $\mathrm{Nd}(\sim 29.1 \%)$ and a higher concentration of $\mathrm{Fe}(\sim 63$ at. \%) [32]. Sasaki et al.

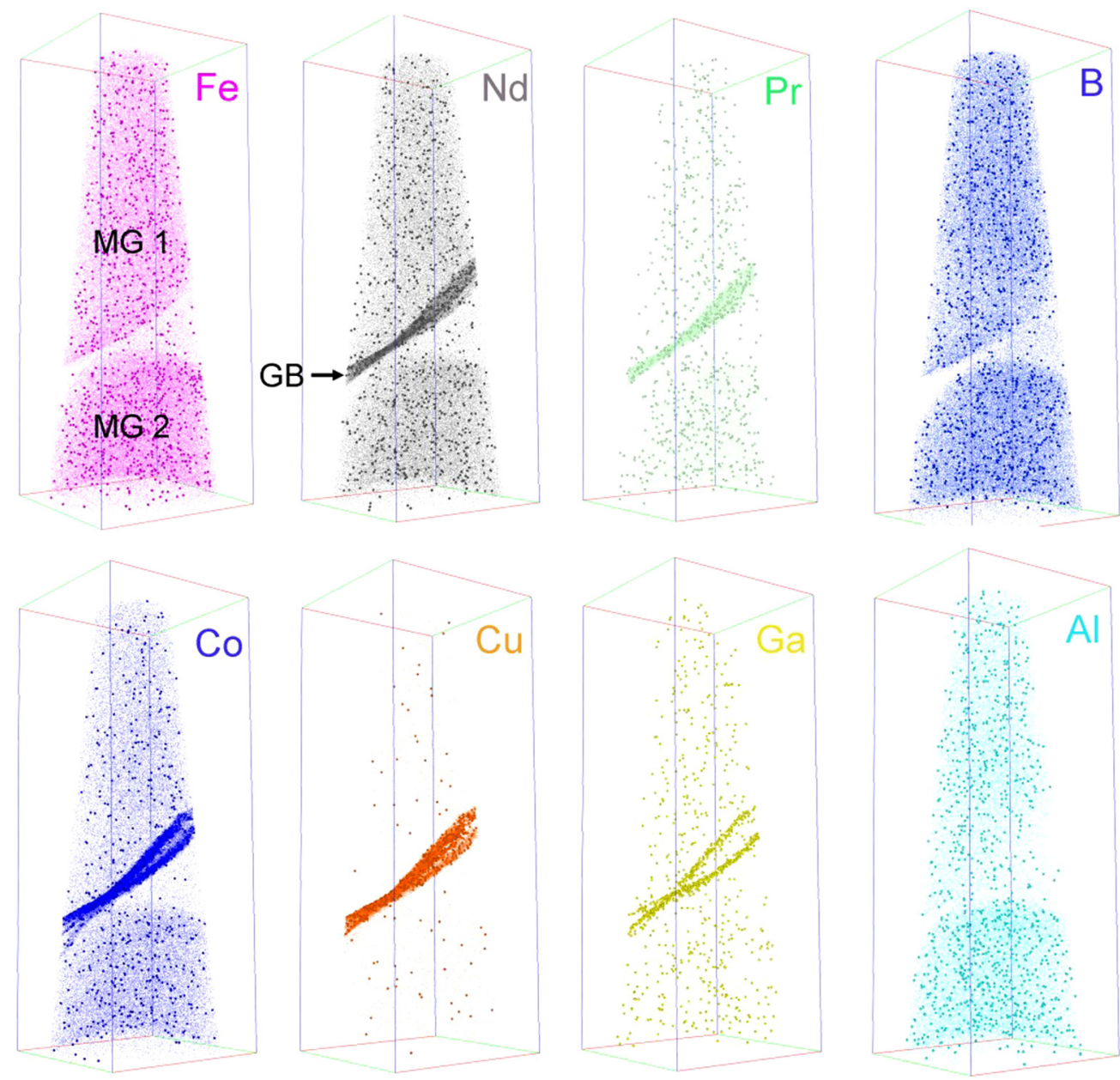

FIG. 3. APT results of an inhomogeneous GB showing $3 \mathrm{D}$ atom distribution maps of $\mathrm{Fe}, \mathrm{Nd}, \mathrm{Pr}, \mathrm{B}, \mathrm{Co}, \mathrm{Cu}, \mathrm{Ga}$, and $\mathrm{Al}$ of the tip. Bounding box dimensions are $\sim 70 \mathrm{~nm} \times 70 \mathrm{~nm} \times 190 \mathrm{~nm}$. 
(a)

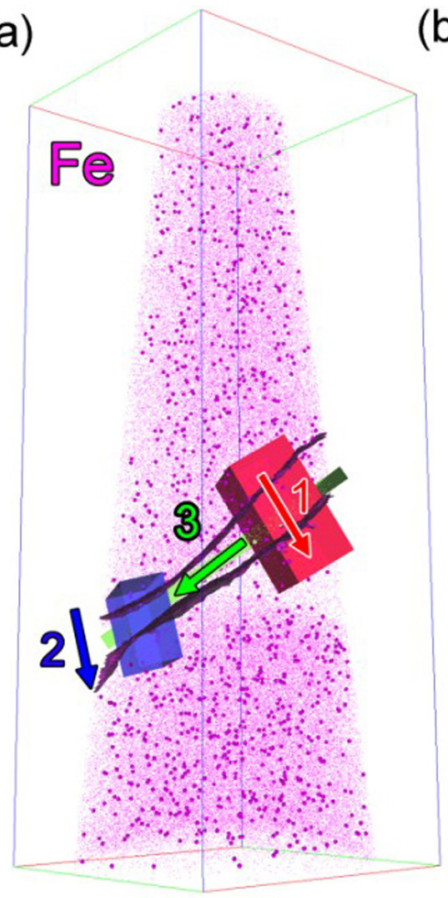

(b)
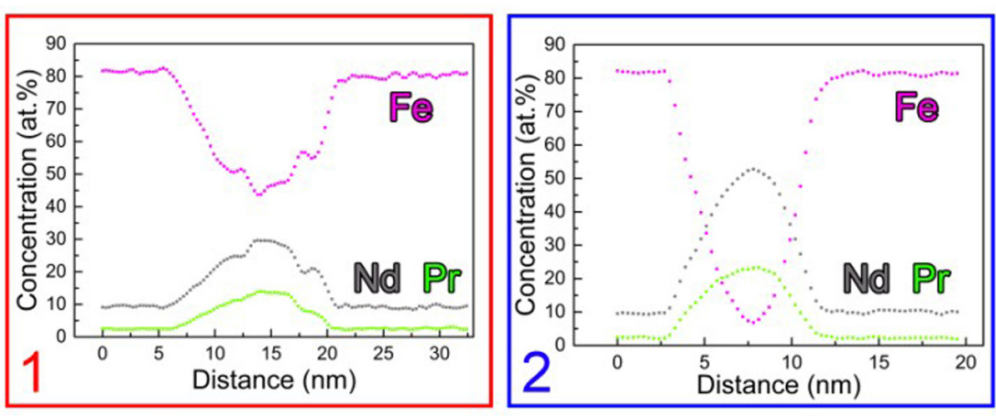

(c)

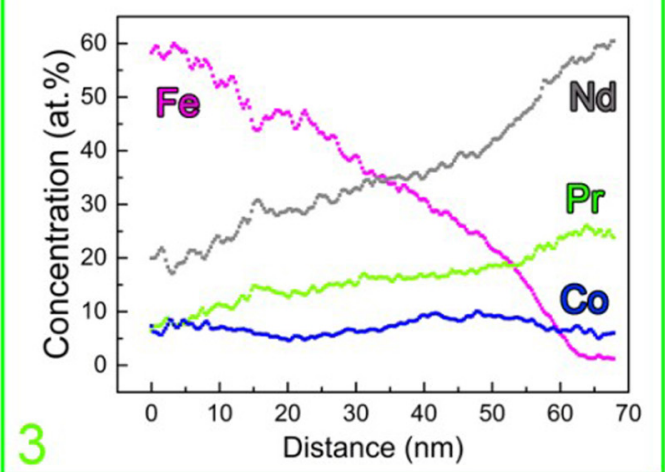

FIG. 4. Enlarged APT results and quantitative compositional analysis. (a) 3D atom distribution map of Fe with isoconcentration surfaces of $\mathrm{Fe}(74.8$ at. \%). (b) 1D concentration profiles of $\mathrm{Fe}, \mathrm{Nd}$, and $\mathrm{Pr}$ of the corresponding red and blue boxes in (a). (c) 1D concentration profiles of Fe, Nd, Pr, and Co of the corresponding green box in (a). Bounding box dimensions are $\sim 70 \mathrm{~nm} \times 70 \mathrm{~nm} \times 190 \mathrm{~nm}$. Supplemental Movie 1 shows the 3D rotation of $\mathrm{Fe}$ atoms [20].

also found that the crystalline Nd-rich GB contains a greater amount of $\mathrm{Nd}+\operatorname{Pr}(\sim 50$ at. \%) and a lower concentration of $\mathrm{Fe}(\sim 40$ at. $\%)$ in a post-sinter-annealed low-carbon $\mathrm{Nd}$ Fe-B permanent magnet [31]. Sepehri-Amin also reported that an amorphous GB contains a lower amount of $\mathrm{Nd}+\mathrm{Pr}$ ( $\sim 33$ at. \%) and a higher concentration of $\mathrm{Fe}+\mathrm{Co}(\sim 64$ at. \%) [11]. From these observations, we inferred that GBs with an Fe concentration greater than 40 at. $\%$ are more likely to be amorphous, while GBs with an Fe concentration less than 40 at. $\%$ are more likely to be crystalline. However, as previously mentioned, the crystalline structures of Nd-rich GBs may easily change during sample preparation and the composition of GBs detected by TEM-energy-dispersive spectroscopy is not accurate due to the small width of the GBs. Given that (1) crystalline structures of GBs are still not well understood, (2) no direct correlation exists between crystalline structures and compositions of GBs, and (3) there is difficulty in observing compositions and crystalline structures of GBs using APT simultaneously, we regarded grain boundaries as amorphous.

Here, Co atoms were regarded the same as $\mathrm{Fe}$ atoms for simplicity because the concentration of Co is only approximately one-tenth of the maximum Fe concentration at the GB. The $M_{S}$ of the GBs $\left[M_{\mathrm{GB}}(\mathrm{MA} / \mathrm{m})\right]$ with various compositions of Fe can be expressed as

$$
M_{\mathrm{GB}}=M_{\mathrm{Nd}_{2} \mathrm{Fe}_{14} \mathrm{~B}} \times \frac{x}{81.9 \text { at. } \%},
$$

where $x$ represents the concentration of $\mathrm{Fe}+\mathrm{Co}$ at the $\mathrm{GB}$ and $M_{\mathrm{Nd}_{2} \mathrm{Fe}_{14} \mathrm{~B}}$ represents $M_{\mathrm{S}}$ of the $\mathrm{Nd}_{2} \mathrm{Fe}_{14} \mathrm{~B}$ matrix phase, which is $1.30 \mathrm{MA} / \mathrm{m}$. The calculated $M_{\mathrm{GB}}$ with 67 at. $\% \mathrm{Fe}$ fits well with the experimental value $(\sim 1.0 \mathrm{~T})$ determined by electron holography [33].

According to the experimental magnetic properties of amorphous alloy films of $\mathrm{Dy}_{x} \mathrm{Fe}_{1-x}$ [34], the $K_{1}$ of the GBs $\left[K_{\mathrm{GB}}\left(\mathrm{MJ} / \mathrm{m}^{3}\right)\right]$ with various compositions of $\mathrm{Fe}$ can be expressed as

$$
K_{\mathrm{GB}}=|36.3 y-27.6| \times 10^{-2},
$$

where $y$ corresponds to the concentration of $\mathrm{Fe}+\mathrm{Co}$ at the GB. The calculated $K_{\mathrm{GB}}$ is one or two orders of magnitude smaller than the $K_{1}=4.5 \mathrm{MJ} / \mathrm{m}^{3}$ of pure $\mathrm{Nd}_{2} \mathrm{Fe}_{14} \mathrm{~B}$ matrix grains.

The relation between the $A$ of the GBs $\left[A_{\mathrm{GB}}(\mathrm{pJ} / \mathrm{m})\right]$ and their Curie temperature $\left(T_{C}\right)$ is shown as [24]

$$
A_{\mathrm{GB}}=\frac{3 k_{B} T_{C}}{2 Z S(S+1)},
$$

where $k_{\mathrm{B}}, Z$, and $S$ correspond to the Boltzmann constant, coordination number, and spin quantum number of the material, respectively. However, measuring the $T_{C}$ of the GBs with various compositions of $\mathrm{Fe}$ remains a challenge.

Here, $9.1 \mathrm{pJ} / \mathrm{m}$ was selected as the reference $A$ for the $67 \mathrm{Fe}$ GB because of the similarity between the $\mathrm{REFe}_{2}$ system and the published $\mathrm{Tb}_{0.3} \mathrm{Dy}_{0.7} \mathrm{Fe}_{2}$ system [35]. Furthermore, $A_{\mathrm{GB}}$ with various compositions of $\mathrm{Fe}$ was calculated using the results recently reported by Sakuma et al. [34]. They reported the magnetism of amorphous $\mathrm{Nd}_{100-x} \mathrm{Fe}_{x}$ films and found that the $\mathrm{Nd}_{100-x} \mathrm{Fe}_{x}$ films exhibit ferromagnetism at room temperature when the $\mathrm{Nd}$ content is less than 70 at. \%. Therefore, we calculated the $A_{\mathrm{GB}}$ based on the magnetic properties obtained in the amorphous $\mathrm{Nd}_{100-x} \mathrm{Fe}_{x}$ system. When the composition 
of Fe at the GB corresponds to zero, the $A_{\mathrm{GB}}, K_{\mathrm{GB}}$, and $M_{\mathrm{GB}}$ are all selected to be zero as a result of the absence of ferromagnetic elements.

\section{Micromagnetic simulations}

To quantify the effects of local composition variation across and along the $\mathrm{GB}$ on the $H_{\mathrm{ci}}$, microstructural observation-based micromagnetic simulations were carried out via the OOMMF platform. Table SII shows that the simulated $H_{\text {ci }}$ values are $5.30,5.30,5.30,5.10,4.20,3.60,3.15,2.85$, and $3.05 \mathrm{~T}$ for the sandwich models containing the $0,10,20,30,40,50,60,67 \mathrm{Fe}$, and inhomogeneous (38.7Fe) GBs, respectively [20]. These results show that the $H_{\mathrm{ci}}$ remains nearly constant when the concentration of $\mathrm{Fe}$ is less than 30 at. \% at the GB, in agreement with the work of Sakuma et al. [34], but the $H_{\mathrm{ci}}$ decreases significantly from 5.10 to $2.85 \mathrm{~T}$ when the composition of $\mathrm{Fe}$ increases from 30 to 67 at. $\%$ at the GB. Strikingly, the simulated $H_{\mathrm{ci}}$ induced by the inhomogeneous GB $(38.7 \mathrm{Fe})$ was determined to be $3.05 \mathrm{~T}$, which is $\sim 27 \%$ smaller than that induced by the homogeneous GB (40Fe). This difference indicates that the observed composition variation along the GB is detrimental to achieving a high $H_{\text {ci }}$. The micromagnetic model shown in Fig. S2(a) is only a small portion of the whole sample [20]; thus, the simulated demagnetization curves in Fig. 5(a) cannot be directly compared to the experimental curves.

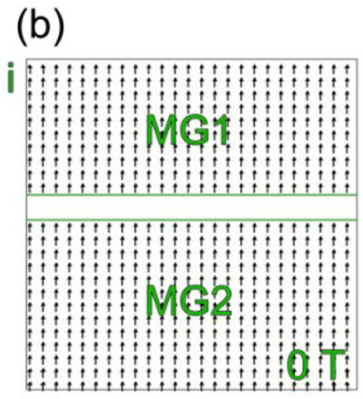

(c)

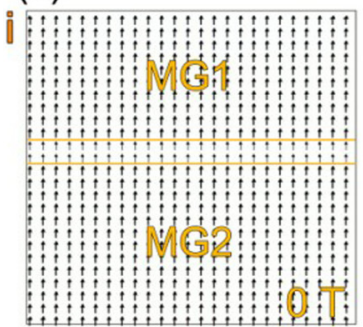

(d)

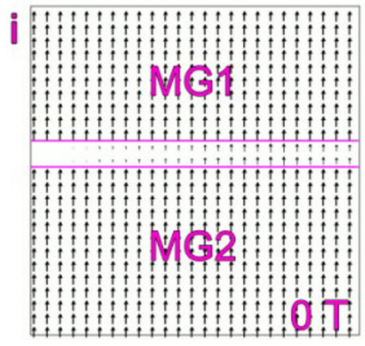

(a)
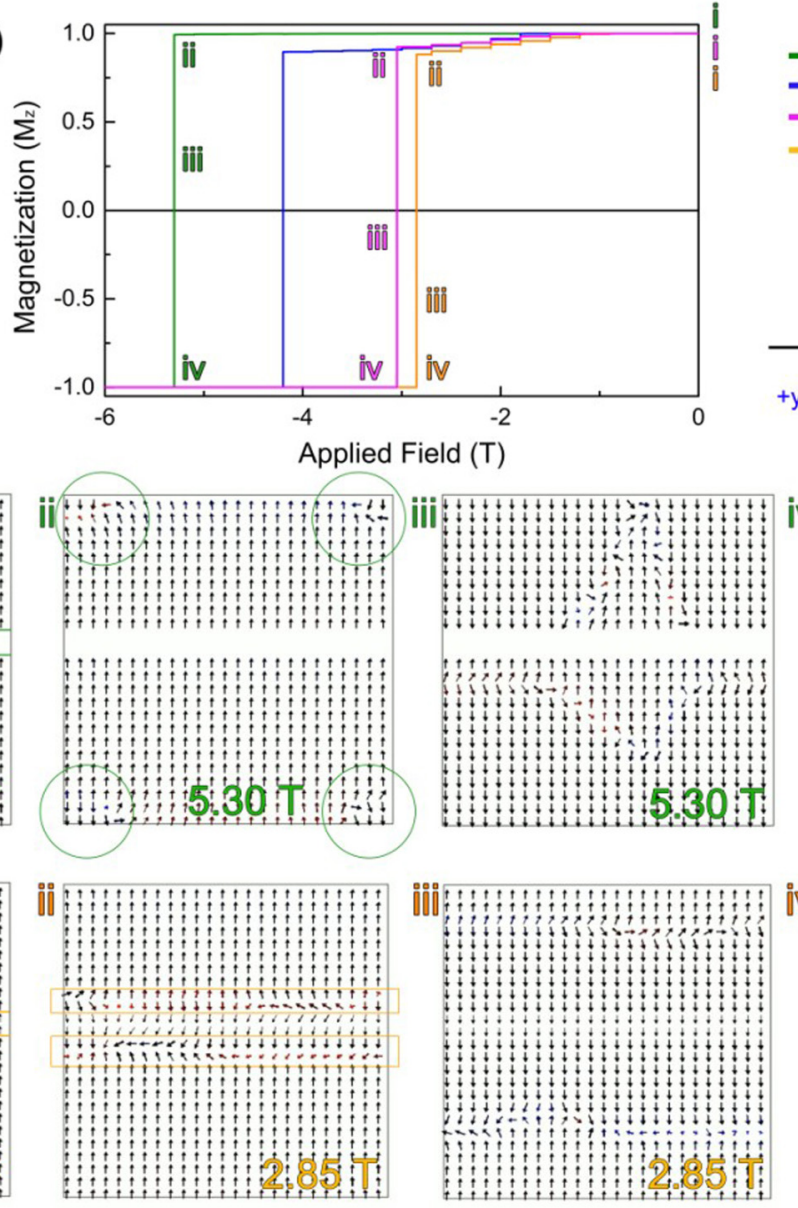
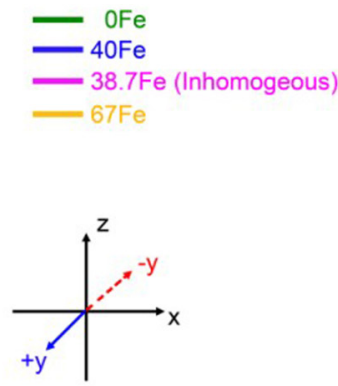
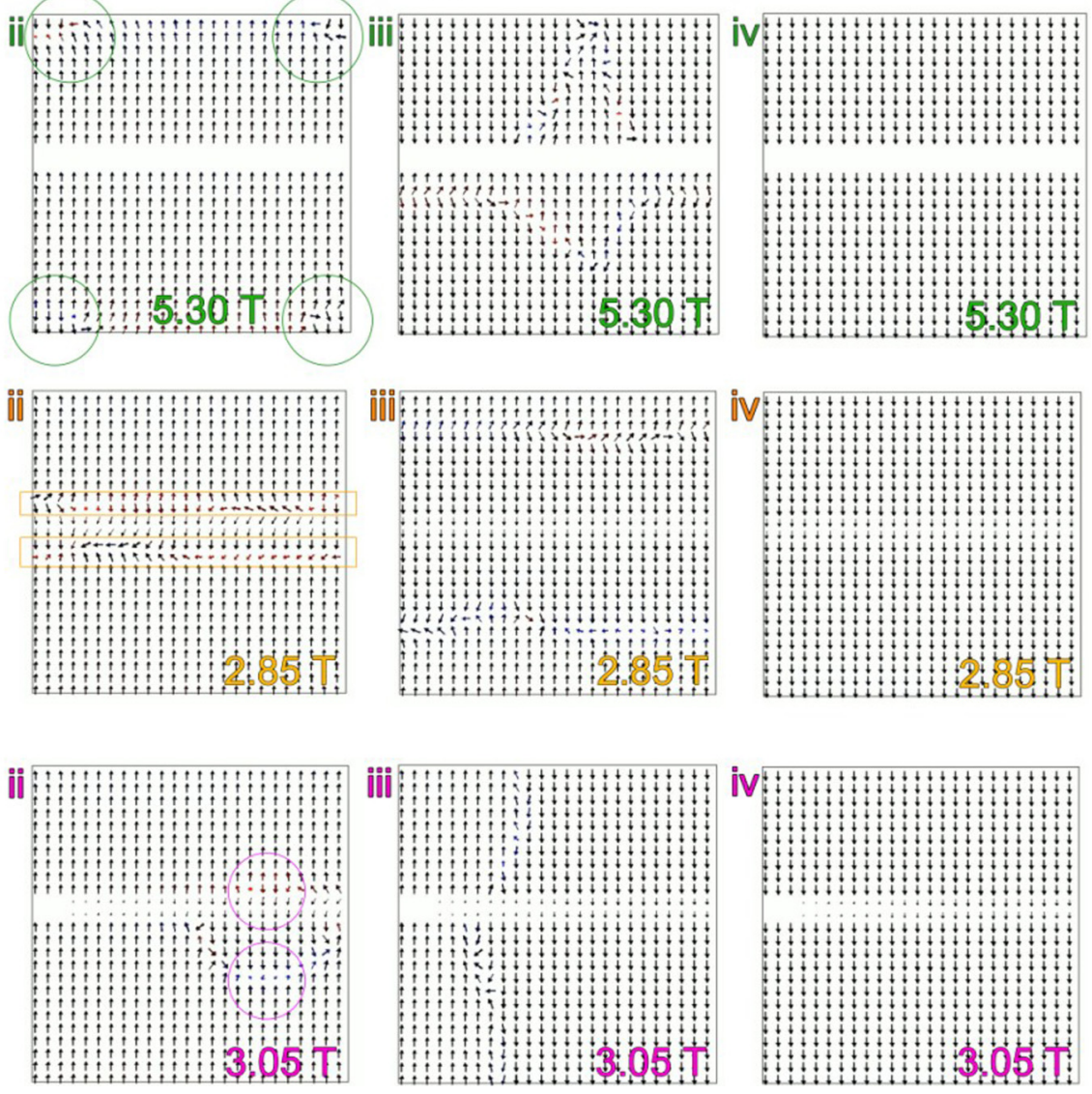

FIG. 5. Micromagnetic simulation results. (a) Simulated demagnetization curves of the sandwich models containing 10-nm-wide GBs with $\mathrm{Fe}$ concentrations of 0,40 , and 67 at. \% and with an inhomogeneous GB with an average Fe composition of $\sim 38.7$ at. \%, in which the concentration of Fe increases from 0 to 67 at. \% gradually and decreases to 50 at. \% in $100 \mathrm{~nm}$, as shown in Fig. S2(b) [20]. Simulated magnetization reversal process of (b) the sandwich model containing MG1, GB (0Fe), and MG2. (c) Sandwich model containing MG1, GB (67Fe), and MG2. (d) Sandwich model containing MG1, inhomogeneous (38.7Fe) GB, and MG2. 

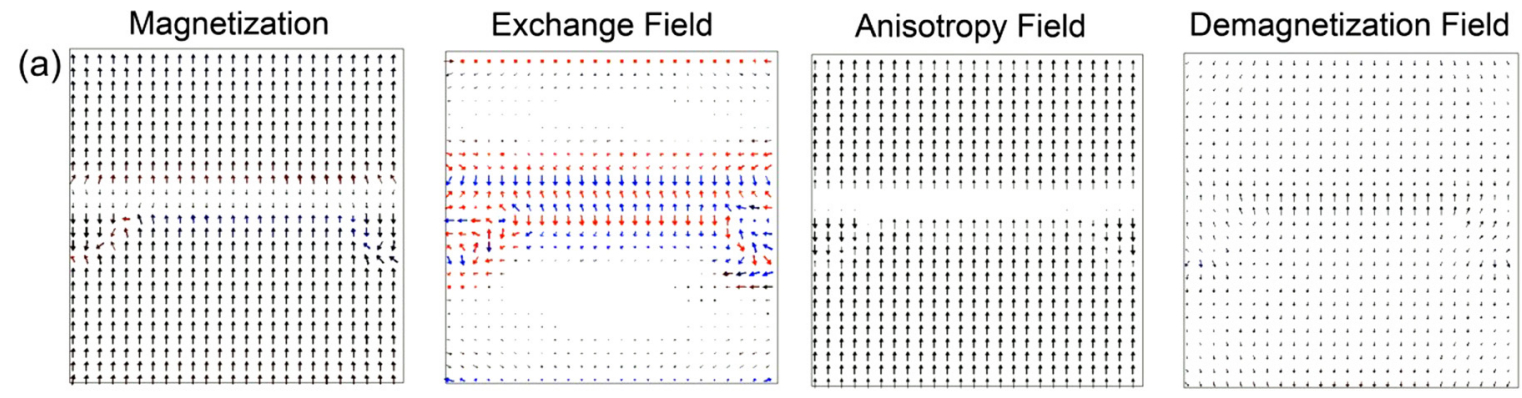

(b)
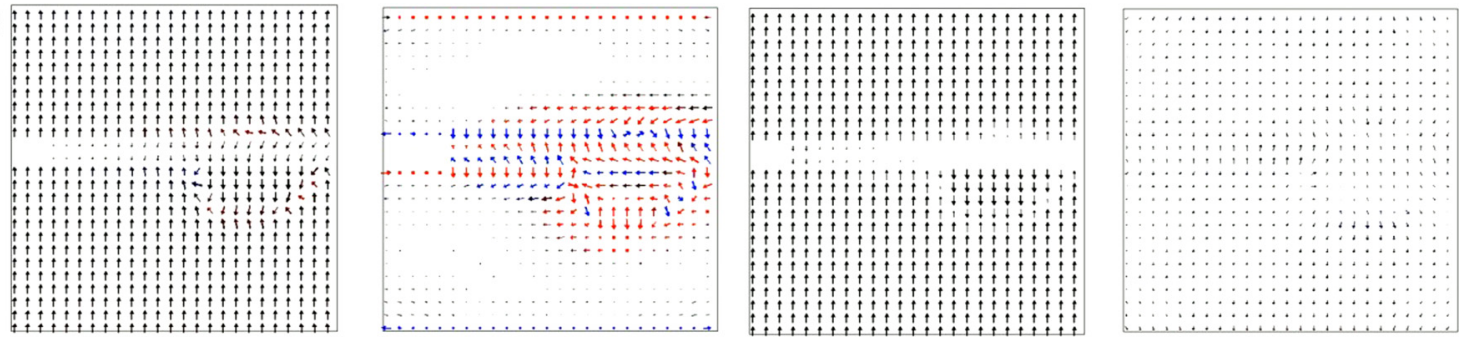

FIG. 6. Micromagnetic simulation results. Calculated magnetization, exchange field, magnetocrystalline anisotropy field, and demagnetization field of the sandwich model consisting of (a) a homogeneous GB with 40 at. \% Fe and (b) an inhomogeneous GB with an average Fe content as high as 38.7 at. $\%$, respectively, at the point of the nucleation process. The scales of the magnetization, exchange field, magnetocrystalline anisotropy field, and demagnetization field of the homogeneous GB are $1.17 \times 10^{6}, 2.29 \times 10^{4}, 5.51 \times 10^{6}$, and $3.35 \times 10^{5} \mathrm{~A} / \mathrm{m}$, respectively. The scales of the magnetization, exchange field, magnetocrystalline anisotropy field, and demagnetization field of the inhomogeneous GB are $1.31 \times 10^{6}, 2.15 \times 10^{4}, 5.51 \times 10^{6}$, and $1.59 \times 10^{6} \mathrm{~A} / \mathrm{m}$, respectively.

To better understand the undesired $H_{\mathrm{ci}}$ degradation resulting from the nanoscale composition variation along the GB, we compared the demagnetization process and corresponding magnetization states at different stages. Figure 5(a) shows the demagnetization curves of the sandwich models containing the $0,40,67 \mathrm{Fe}$, and inhomogeneous $(38.7 \mathrm{Fe}) \mathrm{GBs}$, as revealed by APT. The magnetic moments of the GBs other than that of $0 \mathrm{Fe}$ begin to rotate and reverse under a small external field. As a result, the reverse magnetic moments of these GBs reduce the total magnetization at the beginning, as shown in the simulated demagnetization curves. In the sandwich model with the $0 \mathrm{Fe}$ $\mathrm{GB}$, the reverse magnetic moments nucleate at the corners in MG1 and MG2 because of strong decoupling between the matrix grains and the GB, as shown in the green circles in Fig. 5(b). Once the reverse magnetic domains occur at the corners, magnetic domain walls promptly propagate through the matrix grains, mainly arising from the strong exchange stiffness within the matrix grains. In contrast to the magnetic moments of the $0 \mathrm{Fe} \mathrm{GB}$, those of the $67 \mathrm{Fe} \mathrm{GB}$ begin to reverse under a small external field in the sandwich model with the $67 \mathrm{Fe} \mathrm{GB}$, because the strong exchange coupling between the GB and matrix grains promotes the nucleation of reverse magnetic domains at the interfaces between the GB and the matrix grains. Hence, $H_{\mathrm{ci}}$ is speculated to decrease substantially with the enhanced exchange coupling between the GB and the matrix grains, consistent with previous work $[5,15]$.

Figure 5(d) shows that reverse magnetic moments of the inhomogeneous $(38.7 \mathrm{Fe}) \mathrm{GB}$ nucleate from the interfaces between the matrix grains and GB region with an Fe concentration of $\sim 50-67$ at. $\%$, regardless of the $\sim 40$-nm nonmagnetic regions along the inhomogeneous GB. As a result, the simulated $H_{\mathrm{ci}}$ induced by the inhomogeneous (38.7Fe) GB is very similar to that induced from the homogeneous GB with an average composition of $\sim 67$ at. $\% \mathrm{Fe}$. After the reverse magnetic domains nucleate at such regions, magnetic domain walls promptly propagate through the matrix grains, driven by the strong exchange stiffness within the matrix grains. The nucleation field induced by the inhomogeneous GB (with an average composition of $\sim 38.7$ at. $\% \mathrm{Fe}$ ) is $\sim 27 \%$ smaller than that induced by the homogeneous GB with 40 at. $\% \mathrm{Fe}$, which suggests that the observed composition variation along the GB plays a detrimental role in determining the nucleation field and, thus, the $H_{\text {ci }}$.

Figure 6 shows the calculated magnetization, exchange field, magnetocrystalline anisotropy field, and demagnetization field of the sandwich models with a homogeneous GB with 40 at. $\% \mathrm{Fe}$ and those with an inhomogeneous $\mathrm{GB}$ with an average Fe composition of 38.7 at. \% at the point of nucleation process. Generally, the magnetization reversal process begins with the nucleation of energy-favorable reverse domains that occur preferentially at the grain surface where the magnetic anisotropy is reduced and where the local demagnetizing field is the highest. In the nucleation model of reverse domains, the $H_{\text {ci }}$ of sintered Nd-Fe-B magnets can be expressed as $[4,5,36]$

$$
H_{\mathrm{C}}=\frac{2 K_{1}}{\mu_{0} M_{\mathrm{s}}} \alpha_{K}^{\mathrm{nuc}} \alpha_{\varphi}^{\mathrm{eff}}-N_{\mathrm{eff}} M_{\mathrm{s}},
$$

where $N_{\text {eff }}$ is the local effective demagnetizing factor. $\alpha_{K}^{\text {nuc }}$ and $\alpha_{\varphi}^{\text {eff }}$ correspond to the nucleation effects of the inhomogeneity and misaligned matrix grains, respectively.

First, $\alpha_{K}^{\text {nuc }}$ can be expressed as $[4,5,36]$

$$
\alpha_{K}^{\mathrm{nuc}}=1-\frac{1}{4 \pi^{2}} \frac{\delta_{\mathrm{B}}^{2}}{r_{0}^{2}}\left[-1+\sqrt{1+\frac{4 \Delta K r_{0}^{2}}{A}}\right]^{2},
$$


where $\Delta K, \delta_{\mathrm{B}}$, and $r_{0}$ correspond to the reduction of the $K_{1}$ by $\Delta K$, domain-wall width, and half-width of the planar perturbed inhomogeneous region. The domain-wall width $\left(\delta_{\mathrm{B}}\right)$ can be expressed as [24]

$$
\delta_{\mathrm{B}}=\pi \sqrt{\left(\frac{A}{K}\right)} \approx 5.2 \mathrm{~nm} .
$$

For a certain $r_{0}$,

$$
\alpha_{K}^{\mathrm{nuc}} \propto-\left[-1+\sqrt{1+\frac{4 \Delta K r_{0}^{2}}{A}}\right]^{2} .
$$

Table SII shows that the $K_{1}$ for all GBs (except for the OFe GB) in $\mathrm{RE}_{1-x} \mathrm{Fe}_{x}$ decreases with an increase in $x$ [20]. Consequently, the high content of ferromagnetic elements at the GB increases the $\Delta K$, decreases the $\alpha_{K}^{\text {nuc }}$, and thus decreases the $H_{\mathrm{ci}}$ of the sintered Nd-Fe-B permanent magnet.

Second, $\alpha_{\varphi}^{\text {eff }}$ also plays an equivalent role in controlling the $H_{\mathrm{ci}}$. For an isolated grain, the $\alpha_{\varphi}^{\text {eff }}$ can be expressed as $[4,5,36]$

$$
\alpha_{\varphi}^{\text {eff }}=\frac{1}{\cos \varphi} \frac{1}{\left(1+\tan ^{2 / 3} \varphi\right)^{3 / 2}}\left(1+\frac{2 K_{2}}{K_{1}} \frac{\tan ^{2 / 3} \varphi}{1+\tan ^{2 / 3} \varphi}\right),
$$

where $\varphi$ is the angle between the applied field and the negative $c$ axis. For strongly magnetically coupled grains,

$$
\alpha_{\varphi}^{\text {eff }}=\alpha_{\varphi}^{\min } .
$$

For magnetically isolated grains,

$$
\alpha_{\varphi}^{\mathrm{eff}}=\alpha_{\varphi}^{\mathrm{int}}=\int P(\varphi) \alpha_{\varphi}^{\mathrm{nuc}} d \varphi
$$

where $P(\varphi)$ is the probability distribution function of the misaligned grains. The $\alpha_{\varphi}^{\text {eff }}$ should be in the range between the $\alpha_{\varphi}^{\text {min }}$ and the $\alpha_{\varphi}^{\text {int }}$; therefore, $\alpha_{\varphi}^{\text {eff }}$ can be expressed as $[4,5,36]$

$$
\alpha_{\varphi}^{\mathrm{eff}}=c \alpha_{\varphi}^{\min }+(1-c) \alpha_{\varphi}^{\mathrm{int}}
$$

Figure 6 reveals that the in-plane exchange field promotes the nucleation of reverse magnetic domains near the GB with a higher concentration of ferromagnetic elements, which couples the matrix grains and GBs, increases the value of $c$, and thereby reduces the $H_{\mathrm{ci}}$ of the sintered $\mathrm{Nd}-\mathrm{Fe}-\mathrm{B}$ permanent magnet.

The demagnetization field $\left(-N_{\text {eff }} M_{\mathrm{S}}\right)$, the second term in Eq. (4), also plays a vital role in determining the $H_{\text {ci }}$. Figure 6 and Fig. S4 show that the magnetization of a GB with high concentrations of ferromagnetic elements is remarkably larger than that of a GB with low concentrations of ferromagnetic elements, suggesting that the aforementioned GB with high concentrations of ferromagnetic elements possesses a substantially larger demagnetization field under the circumstance that $N_{\text {eff }}$ remains constant [20]. According to Eq. (4), the $H_{\text {ci }}$ is drastically smaller. The reverse magnetic domains are more likely to nucleate from regions with a low magnetocrystalline anisotropy field, high exchange field, and a high demagnetization field.

Our experimental observation-based micromagnetic simulations provide a more accurate picture of the effects of the GB on the $H_{\text {ci }}$ of a Nd-Fe-B-based system and advance our understanding of the coercivity mechanism of sintered Nd-Fe-B permanent magnets. The $H_{\mathrm{ci}}$ can be improved by optimizing the composition homogenization of GBs with a lower concentration of ferromagnetic elements, such as by grain-boundary diffusion process or by tuning the grain shape of matrix grains.

\section{CONCLUSIONS}

To summarize, our study quantifies composition variation along the grain boundary and its influence on the coercivity of sintered $\mathrm{Nd}-\mathrm{Fe}-\mathrm{B}$ permanent magnets through APT and micromagnetic simulations. Our results reveal that the concentration of ferromagnetic elements (Fe and $\mathrm{Co}$ ) varies dramatically from $\sim 67$ to $\sim 10$ at. $\%$ within $\sim 70 \mathrm{~nm}$ along the grain boundary. Micromagnetic simulations demonstrate that the observed composition variations along the grain boundary are harmful to achieving a high nucleation field and, hence, intrinsic coercivity. These results provide atomic-level insights into the coercivity mechanism of rare-earth permanent magnets, with a methodology offering exciting possibilities for the quantitative analysis and prediction of the composition and magnetic properties of other magnetic materials.

\section{ACKNOWLEDGMENTS}

The authors thank Dr. Patrick Trimby, Dr. Anna Ceguerra, Dr. Takanori Sato, Mr. Adam Sikorski, and Dr. Jacob Warner for their technical support. The authors thank the Australian Microscopy and Microanalysis Research Facility (AMMRF), Australian Centre for Microscopy and Microanalysis (ACMM). This work was partly supported by the Australian Research Council (Grant No. DP150100018).
[1] E. Greinacher, Industrial Applications of Rare Earth Elements (American Chemical Society, Washington DC, 1981), Chap. 1.

[2] D. A. Atwood, The Rare Earth Elements: Fundamentals and Applications (John Wiley \& Sons, New York, 2013).

[3] O. Gutfleisch, M. A. Willard, E. Brück, C. H. Chen, S. Sankar, and J. P. Liu, Adv. Mater. 23, 821 (2011).

[4] H. Kronmüller, K.-D. Durst, and M. Sagawa, J. Magn. Magn. Mater. 74, 291 (1988).

[5] H. Kronmüller, Phys. Status Solidi B 144, 385 (1987).
[6] T. Woodcock, Y. Zhang, G. Hrkac, G. Ciuta, N. Dempsey, T. Schrefl, O. Gutfleisch, and D. Givord, Scr. Mater. 67, 536 (2012).

[7] T. Sasaki, T. Ohkubo, Y. Takada, T. Sato, A. Kato, Y. Kaneko, and K. Hono, Scr. Mater. 113, 218 (2016).

[8] W. Li, T. Ohkubo, T. Akiya, H. Kato, and K. Hono, J. Mater. Res. 24, 413 (2009).

[9] B. Gault, M. P. Moody, J. M. Cairney, and S. P. Ringer, Atom Probe Microscopy (Springer Science \& Business Media, New York, 2012), Vol. 160. 
[10] D. J. Larson, T. Prosa, R. M. Ulfig, B. P. Geiser, and T. F. Kelly, Local Electrode Atom Probe Tomography (Springer, New York, 2013).

[11] H. Sepehri-Amin, T. Ohkubo, T. Shima, and K. Hono, Acta Mater. 60, 819 (2012).

[12] H. Sepehri-Amin, T. Ohkubo, and K. Hono, J. Appl. Phys. 107, 09A745 (2010).

[13] W. Li, H. Sepehri-Amin, T. Ohkubo, N. Hase, and K. Hono, Acta Mater. 59, 3061 (2011).

[14] H. Sepehri-Amin, T. Ohkubo, and K. Hono, Acta Mater. 61, 1982 (2013).

[15] T. Schrefl, H. Schmidts, J. Fidler, and H. Kronmüller, J. Magn. Magn. Mater. 124, 251 (1993).

[16] G. Hrkac, T. G. Woodcock, C. Freeman, A. Goncharov, J. Dean, T. Schrefl, and O. Gutfleisch, Appl. Phys. Lett. 97, 232511 (2010).

[17] D. Suss, T. Schrefl, and J. Fidler, IEEE Trans. Magn. 36, 3282 (2000).

[18] J. Liu, H. Sepehri-Amin, T. Ohkubo, K. Hioki, A. Hattori, T. Schrefl, and K. Hono, Acta Mater. 61, 5387 (2013).

[19] T. Ma, M. Yan, K. Wu, B. Wu, X. Liu, X. Wang, Z. Qian, C. Wu, and W. Xia, Acta Mater. 142, 18 (2018).

[20] See Supplemental Material at http://link.aps.org/supplemental/ 10.1103/PhysRevMaterials.2.054404 for further details on the composition of the sample, sample preparation for APT characterization, quantitative APT analysis, the simulation model, and the simulation results.

[21] D. Saxey, J. Cairney, D. McGrouther, T. Honma, and S. Ringer, Ultramicroscopy 107, 756 (2007).
[22] H. Kronmüller and D. Goll, Scr. Mater. 48, 833 (2003).

[23] M. J. Donahue and D. G. Porter, OOMMF User's Guide, v.1.0, in NIST Interagency Report No. NISTIR 6376 (National Institute of Standards and Technology, Gaithersburg, MD, 1999).

[24] J. M. Coey, Magnetism and Magnetic Materials (Cambridge University Press, Cambridge, England, 2010).

[25] H. Sepehri-Amin, T. Ohkubo, M. Gruber, T. Schrefl, and K. Hono, Scr. Mater. 89, 29 (2014).

[26] H. Chen, Y. Yao, J. A. Warner, J. Qu, F. Yun, Z. Ye, S. P. Ringer, and R. Zheng, Micron 101, 41 (2017).

[27] T. Schrefl, H. Schmidts, J. Fidler, and H. Kronmuller, IEEE Trans. Magn. 29, 2878 (1993).

[28] Y. Shinba, T. Konno, K. Ishikawa, K. Hiraga, and M. Sagawa, J. Appl. Phys. 97, 053504 (2005).

[29] W. Mo, L. Zhang, Q. Liu, A. Shan, J. Wu, and M. Komuro, Scr. Mater. 59, 179 (2008).

[30] W. Li, T. Ohkubo, and K. Hono, Acta Mater. 57, 1337 (2009).

[31] T. Sasaki, T. Ohkubo, Y. Une, H. Kubo, M. Sagawa, and K. Hono, Acta Mater. 84, 506 (2015).

[32] T. Sasaki, T. Ohkubo, and K. Hono, Acta Mater. 115, 269 (2016).

[33] T.-S. Chin, C.-H. Lin, H. Bai, Y. Huang, K. Cheng, and S. Huang, IEEE Trans. Magn. 28, 2587 (1992).

[34] A. Sakuma, T. Suzuki, T. Furuuchi, T. Shima, and K. Hono, Appl. Phys. Express 9, 013002 (2015).

[35] D. B. Gopman, J. W. Lau, K. P. Mohanchandra, K. Wetzlar, and G. P. Carman, Phys. Rev. B 93, 064425 (2016).

[36] H. Kronmüller, K.-D. Durst, S. Hock, and G. Martinek, J. Phys., Colloq. 49, C8-623 (1988). 